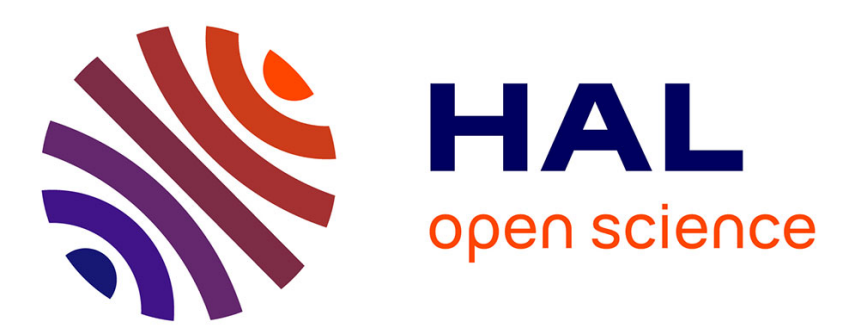

\title{
Modelling wafer bow in siliconpolycrystalline CVD diamond substrates for GaN-based devices
}

\author{
M J Edwards, C R Bowen, D W E Allsopp, a C E Dent
}

\section{To cite this version:}

M J Edwards, C R Bowen, D W E Allsopp, a C E Dent. Modelling wafer bow in siliconpolycrystalline CVD diamond substrates for GaN-based devices. Journal of Physics D: Applied Physics, 2010, 43 (38), pp.385502. 10.1088/0022-3727/43/38/385502 . hal-00569715

\section{HAL Id: hal-00569715 https://hal.science/hal-00569715}

Submitted on 25 Feb 2011

HAL is a multi-disciplinary open access archive for the deposit and dissemination of scientific research documents, whether they are published or not. The documents may come from teaching and research institutions in France or abroad, or from public or private research centers.
L'archive ouverte pluridisciplinaire HAL, est destinée au dépôt et à la diffusion de documents scientifiques de niveau recherche, publiés ou non, émanant des établissements d'enseignement et de recherche français ou étrangers, des laboratoires publics ou privés. 


\title{
Modelling Wafer Bow in Silicon-Polycrystalline CVD Diamond Substrates for GaN-based Devices
}

\author{
M J Edwards ${ }^{1 *}$, C R Bowen ${ }^{1}$, D W E Allsopp ${ }^{2}$ and A C E Dent ${ }^{1}$ \\ ${ }^{1}$ Department of Mechanical Engineering, University of Bath, BA2 7AY. \\ ${ }^{2}$ Department of Electronics and Electrical Engineering, University of Bath, BA2 7AY. \\ *Corresponding email address: m.j.edwards2@ bath.ac.uk
}

\begin{abstract}
Composite silicon-polycrystalline CVD diamond wafers are potential substrates for GaN-based devices for use in harsh environments due to their high thermal conductivity and chemical stability. When cooled from a typical diamond deposition temperature of approximately $800^{\circ} \mathrm{C}$ to $25^{\circ} \mathrm{C}$ wafer bowing arises from a mismatch in the coefficients of thermal expansion of silicon and polycrystalline diamond. In this paper $100 \mathrm{~mm}$ diameter silicon-polycrystalline diamond wafers have been modelled using ANSYS finite element software to investigate their bowing behaviour as a function of temperature and geometry. The maximum bow of a wafer occurred where the thicknesses of both the silicon and polycrystalline diamond layers was almost identical; this has been confirmed using analytical methods. Strategies are discussed for reducing wafer bow.
\end{abstract}

Keywords: Finite-element, CVD diamond, GaN, Silicon, Modelling.

PACS: 62.20.-x:Mechanical properties of solids, 62.20.D-: Elasticity, 68.55.jd:

Thickness, 68.65.Ac: Multilayers

\section{Introduction}

Gallium Nitride $(\mathrm{GaN})$ is a robust material that is stable at temperatures up to $1000^{\circ} \mathrm{C}$, has a wide bandgap of $3.4 \mathrm{eV}$ and a Young's modulus $\left(\mathrm{E}_{<0001>}\right)$ of $\sim 150 \mathrm{GPa}$ at room temperature $[1,2]$. These properties make it suitable for use in harsh environments. The wide band gap and high breakdown field of GaN make it suitable for power electronic devices, from which heat needs to be extracted for optimum performance. In order to create a viable $\mathrm{GaN}$ sensor, high power diode or transistor, a suitable substrate is required to support the device in harsh operating conditions. Silicon-polycrystalline diamond substrates are potentially advantageous, since both materials have high melting points, good mechanical and chemical stability. Polycrystalline diamond is also an excellent thermal conductor, with a conductivity of $2270 \mathrm{~J} / \mathrm{Kms}$ at $20^{\circ} \mathrm{C}$, and has a Young's modulus of $1050 \mathrm{GPa}$ at the same temperature.

Crystalline silicon provides a substrate for the growth of the polycrystalline diamond and, if thinned to a few microns, provides a crystalline base from which epitaxial $\mathrm{GaN}$ can be grown. The (111) orientated silicon is the preferred substrate orientation for epitaxial GaN growth, although growth on $\mathrm{Si}(100)$ and $\mathrm{Si}(110)$ is also possible [3, 4]. However, silicon-polycrystalline wafers are observed to bow when the substrate is cooled from the typical CVD diamond growth temperature $\left(\sim 700-800^{\circ} \mathrm{C}\right)[5,6]$ to room temperature $\left(25^{\circ} \mathrm{C}\right)$. One cause of the bowing is a mismatch in the coefficients of thermal 
expansion (CTE), $\alpha$, between silicon and polycrystalline diamond, which are shown in Figure 1 along with CTE data for GaN. At low temperatures the CTE of diamond is lower than silicon while at higher temperatures the diamond CTE is higher than silicon. The aim this work is to use finite-element simulations to model the mechanical behaviour of silicon-polycrystalline diamond wafers, and to predict their bowing behaviour as a function of temperature and geometry. This work shows that the bow of a $100 \mathrm{~mm}$ diameter wafer is predicted to exceed $1 \mathrm{~mm}$; which will render processing of devices via photolithography very difficult. For example, the requirements for modern semiconductor products are a wafer bow of $<40 \mu \mathrm{m}$ for wafers of $300 \mathrm{~mm}$ diameter [6].

This paper will highlight the modelling techniques explored in this work, an analytical model based on thermal stresses in bi-layers, and a finite element (FE) model. The temperature dependent coefficients of thermal expansion (CTE), $\alpha$, Poisson's ratios, $v$, and Young's modulii, $E$, of both silicon and polycrystalline diamond were used in the model. Initially, a linear average of the temperature dependent material properties was calculated from 25 to $800^{\circ} \mathrm{C}$, since the analytical method assumed that $\alpha, v$ and $E$ were independent of temperature. The temperature independent properties were also used in the FE model to validate the boundary conditions and geometry used, through comparison with the analytical results. Once the FE model was validated, it was extended to include the influence of temperature dependent material properties. Issues relating to (i) the presence of a columnar diamond structure leading and anisotropy of the film and (ii) changes in silicon orientation from (111) to (100) are discussed.

\section{Material Properties of Polycrystalline CVD Diamond and Silicon}

Materials properties were collected to determine the key material properties of silicon and polycrystalline diamond. Both materials feature a cubic crystal structure and hence require three stiffness coefficients, $\left(c_{11}, c_{12}, c_{44}\right)$ to describe the elastic response. Polycrystalline diamond consists of randomly orientated crystalline grains, making it approximately isotropic at the macroscopic level [11-13], although a columnar structure at larger film thicknesses could also be present [14]. At this stage both materials were assumed isotropic for simplicity; texturing of the diamond film will be discussed later in the paper. Consequently, only the Young's modulus, $E$, and x-y Poisson's ratio, $v$, are required for both materials, rather than the full stiffness matrix. This assumption aided the development of a simple analytical model, discussed in section 3, where all materials in the bi-layered wafer system are treated as isotropic. The CTE, $\alpha$, of both silicon and diamond is isotropic owing to its crystallographic structure, such that $\alpha_{x}=\alpha_{y}=\alpha_{z} .[15,16]$

\subsection{Material Properties of Polycrystalline CVD Diamond}

For polycrystalline diamond, without columnar grains [14], the material consists of grains of randomly orientated diamond material with little variation in the elastic modulus along any direction. Sources [1112] report relatively low elastic coefficients for polycrystalline diamond due to imperfections and impurities. Furthermore, the experimental data of Szuecs et al [13] shows that the Young's modulus of CVD polycrystalline diamond is not affected by the grain size if the defect density is constant. Modern growth techniques now used to grow polycrystalline diamond produce significantly reduced defect densities and its Young's modulus can approach that of optical grade diamonds [13]. The values for the elastic modulii of diamond at room temperature were taken from McSkimin and Andreatch [17] a commonly cited source and are quoted in table 1, although the potential influence of the variation in the elastic modulus is also discussed later in the paper.

Table 1. Elastic modulii of diamond at room temperature [17].

\begin{tabular}{cc}
\hline Modulus & $(\mathrm{GPa})$ \\
\hline$c_{11}$ & 1079 \\
$c_{12}$ & 124
\end{tabular}


The Young's modulus and Poisson's ratio at room temperature $\left(25^{\circ} \mathrm{C}\right)$ for diamond were calculated using the elastic modulii values in table 1 and standard formulae [17] to be $E_{R T}=1050 \mathrm{GPa}$ and $v=0.1$. However, it has been found that the Young's modulus of CVD diamond degrades faster with respect to temperature than predicted in McSkimin and Andreatch [17] due to the presence of contaminants. It has been shown experimentally by Szuecs et al [13] that the variation of Young's modulus $(E)$ with temperature, $T$, of CVD diamond undergoes a linear regression of the form

$$
E=E_{R T}\left[1+c_{T}(T-25)\right]
$$

where the temperature coefficient, $c_{T}$, has an average value of $-1.027 \times 10^{-4} \mathrm{~K}^{-1}$. Using a room temperature Young's modulus, $E_{R T}$ [17], equation (1), and the average value for $c_{T}$ from [13], the temperature dependent Young's modulii for polycrystalline CVD diamond were calculated and are shown in table 2. The Poisson's ratio is assumed constant between -100 and $1000^{\circ} \mathrm{C}$.

Table 2. Elastic and thermal properties of CVD diamond used in the model $[7,8,13,18]$.

\begin{tabular}{cllc}
\hline Temp $\left({ }^{\circ} \mathrm{C}\right)$ & $E(\mathrm{GPa})$ & $v^{[17]}$ & $A\left(10^{-6} \mathrm{~K}^{-1}\right)^{[7,8]}$ \\
\hline-100 & 1062 & 0.1 & 0.4 \\
0 & 1052 & 0.1 & 0.8 \\
20 & 1050 & 0.1 & 1 \\
25 & 1050 & 0.1 & 1 \\
50 & 1048 & 0.1 & 1.4 \\
100 & 1043 & 0.1 & 1.7 \\
200 & 1034 & 0.1 & 2.3 \\
300 & 1025 & 0.1 & 3.1 \\
400 & 1015 & 0.1 & 3.7 \\
500 & 1006 & 0.1 & 4.0 \\
600 & 997 & 0.1 & 4.3 \\
700 & 988 & 0.1 & 4.5 \\
800 & 978 & 0.1 & 4.6 \\
900 & 969 & 0.1 & 4.8 \\
1000 & 960 & 0.1 & 4.9 \\
\hline
\end{tabular}

\subsection{Material Properties of (111) Silicon}

While (100) orientated silicon can be used for diamond on silicon [19] this work initially considers (111) orientation silicon since it is for c-plane GaN epitaxial growth [20]. The elastic modulii of isotropic silicon and their temperature dependence are determined using the approach of Varshni [21], which is derived from the Einstein model for a solid and are given by

$$
c_{i j}=c_{i j}^{0}-u /\left(e^{t / T}-1\right)
$$

where $u$ and $t$ are fitting parameters and $c_{i j}{ }^{0}$ is the value for the elastic constant at $0 \mathrm{~K}$. The $0 \mathrm{~K}$ elastic modulii values are based on the work in McSkimin [22], who measured the elastic modulii between 78 and $300 \mathrm{~K}$ using ultrasonic wave techniques. The elastic modulii $0 \mathrm{~K}$ values and fitting constants used in this work are shown in table 3. 
Table 3. 0K Elastic modulii and fitting constants of silicon [21].

\begin{tabular}{cccc}
\hline$c_{i j}$ & $c_{i j}{ }^{0}(\mathrm{GPa})$ & $u(\mathrm{GPa})$ & $t(\mathrm{~K})$ \\
\hline$c_{11}$ & 167.5 & 5.33 & 407.0 \\
$c_{12}$ & 65.0 & 2.31 & 334.8 \\
$c_{44}$ & 80.0 & 2.25 & 497.4 \\
\hline
\end{tabular}

As the silicon is (111) orientated in the model, the compliance coefficients, $s_{i j}$, need to be calculated from the elastic modulii from the standard formulae in [23]. Using the compliance coefficients calculated from [11], the Young's modulus, $E$ (111), and Poisson's ratio, $v$ (111), of silicon along the (111) plane is calculated via

$$
\begin{aligned}
& E(111)=\frac{4}{2 s_{11}+2 s_{12}+s_{44}} \\
& v(111)=\frac{2 s_{11}+10 s_{12}-s_{44}}{6 s_{11}+6 s_{12}+3 s_{44}}
\end{aligned}
$$

The derivation of equations (3) and (4) can be found in Cho [23]. Equations (3) and (4) can also be derived using the compliance coefficients for rotated axes in cubic crystals from Wortman and Evans [24]. Although (111) silicon has an independent shear modulus, it has been treated as an isotropic material [23]. Initial exploration of this problem has indicated that the error caused by assuming that (111) silicon is isotropic is less than $1 \%$ and will have little effect on the model results shown in this paper. Therefore, it has been assumed that (111) silicon has isotropic mechanical properties. The temperature dependent Young's modulii and Poisson's ratios, calculated using equations (3) and (4), are presented in table 4 for (111) silicon alongside its temperature dependent CTEs [9].

Table 4. Elastic and thermal properties of silicon used in the model [7,9, 21-24].

\begin{tabular}{cccc}
\hline Temp $\left({ }^{\circ} \mathrm{C}\right)$ & $E(111)(\mathrm{GPa})$ & $v(111)$ & $\alpha\left(10^{-6} \mathrm{~K}^{-1}\right)^{[9]}$ \\
\hline-173 & 170.3 & 0.26 & -0.509 \\
-73 & 169.8 & 0.26 & 1.453 \\
7 & 169.2 & 0.26 & 2.392 \\
27 & 169.0 & 0.26 & 2.568 \\
127 & 168.1 & 0.26 & 3.212 \\
227 & 167.1 & 0.26 & 3.594 \\
327 & 166.0 & 0.26 & 3.831 \\
427 & 165.0 & 0.26 & 3.987 \\
527 & 163.9 & 0.26 & 4.099 \\
627 & 162.8 & 0.26 & 4.185 \\
727 & 161.8 & 0.26 & 4.264 \\
827 & 160.7 & 0.26 & 4.322 \\
927 & 159.6 & 0.25 & 4.380 \\
1027 & 158.5 & 0.25 & 4.438 \\
1127 & 157.4 & 0.25 & 4.496 \\
1227 & 156.3 & 0.25 & 4.554 \\
\hline
\end{tabular}


The data in table 4 shows that (111) silicon has a high degree of thermal stability, with only a small decrease in both $E$ and $v$ as the temperature increases.

\section{Analytical Model}

The lack of detailed bowing measurements of the bowing of polycrystalline diamond - silicon composite wafers means that a method of confirming the prediction of the finite element model is required. A comparison is made with the predictions of an analytical model based on the theory of thermal stresses in elastic multilayer structures described by Hsueh [25] and figure 2 .

\subsection{Analytical model with temperature independent properties.}

In the analytical method, it is assumed that the silicon-polycrystalline diamond wafer is $100 \mathrm{~mm}$ in diameter, perfectly elastic and cooled to room temperature $\left(25^{\circ} \mathrm{C}\right)$ from a growth temperature of $800^{\circ} \mathrm{C}$ $\left(\Delta \mathrm{T}=775^{\circ} \mathrm{C}\right)$; this is similar to the deposition temperatures used in Zimmer et al [5]. Due to a mismatch in CTEs the film and substrate contract by differing amounts (figure $2 b$ ) and since the strain in the wafer is constrained it bows due to asymmetric stresses (figure 2c). If the silicon substrate (with a higher CTE) contracts to a greater degree than the diamond, the silicon is under tension while the diamond film is under compression. The stress-strain distribution in such a bi-layered system is dependent on three parameters. These parameters are the uniform strain component, $c$, the bending axis, $t_{b}$, and the radius of curvature, $r$. The stress-strain parameters can be determined through the total strain in the system, $\varepsilon$, the normalized biaxial stresses, $\sigma_{S b}$ and $\sigma_{F b}$, and three sequential boundary conditions discussed in [25]. Firstly, the model assumes that both silicon and polycrystalline diamond have isotropic properties to simplify the derivation. Secondly, as the wafer undergoes biaxial strain it has planar geometry and the Young's modulii of the substrate (silicon) and film (diamond) are adjusted according to [25] such that

$$
\begin{gathered}
E_{S b}=\frac{E_{S}}{1-v_{s}} \\
E_{F b}=\frac{E_{F}}{1-v_{F}}
\end{gathered}
$$

where $E_{S}$ and $E_{F}$ are the Young's modulii of the film and substrate respectively, with $E_{S b}$ and $E_{F b}$ being the adjusted Young's modulii that take the biaxial strain of the system into account. The Poisson's ratios of the film and substrate are $v_{F}$ and $v_{S}$ respectively.

The normalized biaxial stresses on film and substrate materials and the total strain in the system are

$$
\begin{gathered}
\varepsilon=c+\frac{z-t_{b}}{r} \\
\sigma_{S b}=E_{S b}\left(\varepsilon-\alpha_{S} \Delta T\right) \\
\sigma_{F b}=E_{F b}\left(\varepsilon-\alpha_{F} \Delta T\right)
\end{gathered}
$$

Using boundary conditions derived from equations (7) - (9) [25], it is possible to derive the universal strain component, $c$, the bending axis, $t_{b}$, and the radius of curvature, $r$, for an elastic bi-layered strip. 


$$
\begin{gathered}
c=\frac{\left(E_{S b} t_{S} \alpha_{S}+E_{F b} t_{F} \alpha_{F}\right) \Delta T}{E_{S b} t_{S}+E_{F b} t_{F}} \\
t_{b}=\frac{E_{F b} t_{F}^{2}-E_{S b} t_{S}^{2}}{2\left(E_{S b} t_{S} E_{F b} t_{F}\right)} \\
r=\frac{E_{S b} t_{S}^{2}\left(2 t_{S}+3 t_{b}\right)+E_{F b} t_{F}^{2}\left(2 t_{F}-3 t_{b}\right)}{3\left[E_{S b} t_{S}^{2}\left(c-\alpha_{S} \Delta T\right)-E_{F b} t_{F}^{2}\left(c-\alpha_{F} \Delta T\right)\right.}
\end{gathered}
$$

With $r$ derived it is possible to calculate the bow, $b$, of the wafer using geometry.

\subsection{Determining Wafer Bow from the Radius of Curvature.}

It is possible using simple geometry to determine the bow, $b$, of the silicon-polycrystalline diamond wafer from its radius of curvature, $r$, and radius of the wafer, $R_{W}$. Wafer bow is defined as the change in displacement in the $\mathrm{z}$ direction, shown in figure 3. It is assumed in the model that the wafer is a perfect circle and therefore has rotational symmetry; this means that using a two-dimensional derivation as shown in figure 2 is acceptable. Figure 3 shows the geometry used to derive the resultant equation for determining wafer bow and is:

$$
b=-r \pm \sqrt{r^{2}-R_{W}^{2}\left(\frac{\cos \left(\frac{3 R_{W}}{4 \pi r}\right)}{\left.\cos \left(\frac{R_{W}}{2 \pi r}\right)\right)^{2} \cos ^{2}\left(\frac{R_{W}}{4 \pi r}\right)}\right.}
$$

The sign of the " \pm " is dependent on the sign of the radius of curvature, with a minus sign used for a negative radius of curvature and vice versa.

It is possible to simplify equation (13) further when $\theta \rightarrow 0$, when $b$ becomes

$$
b=-r-\sqrt{r^{2}-R_{W}^{2}}
$$

This small angles approximation holds when the bow is below $3 \mathrm{~mm}$ and the radius of curvature is negative.

\section{Finite Element (FE) Model}

The FE model was developed using commercial software (ANSYS 11.0), chosen for its multi-physics capacity that allows the temperature dependent behaviour of the structural properties to be considered. The model assumes that the wafer obeys simple linear elasticity with no plastic deformation of the substrate or the presence of intrinsic stress within the diamond film. Before a FE model can be used it needs to be tested against either measured data or a rigorous analytical method. There is limited experimental data on the bow of silicon-polycrystalline diamond wafers [5,6], therefore the only reliable method of validating the model was to compare its results with those of the above analytical approach in which the wafer is assumed to act as an elastic bi-layered strip under biaxial strain. This is a reasonable assumption because the wafer has rotational symmetry, and can be assumed elastic over a wide range of stresses. Zimmer et al. [5,6] indicated that a non-uniform temperature distribution across the siliconpolycrystalline diamond wafer during processing leads to asymmetric stress distributions that can distort 
the wafer to a saddle shape. However, using hot filament CVD technology Zimmer et al. produced a thermally uniform wafer [5], with a radial stress profile, and more symmetric wafer bow. One of the disadvantages of the analytical method is that it assumes that the stiffness and CTEs do not change with respect to temperature.

Figure 4(a) shows schematically the structure modelled using finite element analysis; it consists of a top film layer of polycrystalline CVD diamond and a bottom substrate layer of (111) silicon and is modelled in ANSYS using PLANE13 elements [26]. For efficient computation, axisymmetric boundary conditions were used, as shown in figure 4(a). The PLANE13 element type was chosen because it is fully axisymmetric and its degrees of freedom include the two required spatial dimensions and temperature. The wafer was clamped at the bottom edge to provide a simple measure of wafer bow.

The bow of the wafer was calculated for different thicknesses of the silicon substrate, $t_{s}$, between 1000 and $3 \mu \mathrm{m}$. The underlying interest in composite polycrystalline diamond - silicon wafers is their potential as substrates with superior heat sinking for high power $\mathrm{GaN}$ electronic and optoelectronic devices, exploiting the superior thermal conduction of polycrystalline diamond. As such, a thick silicon layer is undesirable as it would impair the performance of any potential device. Thus, the primary purpose of the FE model is to model the bowing behaviour of the wafer as it is cooled and subsequently mechanically thinned down to the required silicon thickness. Referring to figures 4(a) and 4(b), the bow is defined as the change in displacement in the z-direction from the bottom right-hand corner of the wafer with respect to the origin.

\section{Comparison of FE Model and the Analytical Method}

The main limitation of the analytical model is that it assumes that the Young's modulii, Poisson's ratios and CTEs of both polycrystalline CVD diamond and silicon do not vary with temperature, which could lead to errors in predicted bow. However, it is possible to include the temperature dependence of properties using a linear average of the temperature dependent material properties from table 2 and table 4. The temperature independent averages of the Young's modulii, Poisson's ratios and CTEs of silicon and polycrystalline diamond between 25 and $800^{\circ} \mathrm{C}$ used in the analytical and $\mathrm{FE}$ model are in table 5 . The higher linear average CTE of silicon, compared to diamond, leads to the wafer bowing as in figure $2 \mathrm{c}$ with the silicon under tension and diamond in compression.

Table 5. Temperature independent (linearly averaged) material properties of silicon and polycrystalline CVD diamond between the temperatures of 25 and $800^{\circ} \mathrm{C}$.

\begin{tabular}{cccc}
\hline Material & $E\left(25-800^{\circ} \mathrm{C}\right) /$ & $v\left(25-800^{\circ} \mathrm{C}\right)$ & $\alpha\left(25-800^{\circ} \mathrm{C}\right) /$ \\
& $\mathrm{GPa}$ & & $10^{-6} \mathrm{~K}^{-1}$ \\
\hline Silicon & 165.5 & 0.26 & 3.45 \\
Polycrystalline Diamond & 1014.0 & 0.1 & 2.8 \\
\hline
\end{tabular}

In the example used in this work the bowing behaviour of a $100 \mathrm{~mm}$ diameter composite siliconpolycrystalline CVD diamond wafer, with a polycrystalline CVD diamond thickness $t_{F}=100 \mu \mathrm{m}$ and temperature independent material properties from table 5, have been calculated using the analytical and FE method. The thickness of the silicon substrate, $t_{S}$, was varied between $3 \mu \mathrm{m}$ and $1000 \mu \mathrm{m}$ and the wafer bow was measured after cooling the composite substrate from 800 to $25^{\circ} \mathrm{C}\left(\Delta \mathrm{T}=775^{\circ} \mathrm{C}\right)$. Figure 5 shows a comparison of the predicted wafer bow found from the FE model and the analytical model. The bowing values calculated from the analytical model are shown by the continuous line, whilst those found from the temperature independent FE model are shown by the square data points ( $(\mathbf{)})$.

Excellent agreement is observed between the analytical and temperature independent FE models, indicating mutual consistency and implies that the boundary conditions and mesh densities employed in the FE approach are appropriate. Good agreement is expected because both models use identical material 
properties and have identical geometries. It is of interest to note that both models predict that the bowing is a maximum when the thickness of the silicon almost equals that of the polycrystalline diamond film $\left(t_{S}\right.$ $\sim t_{F}$ ), with the bow then falling as the silicon thickness decreases to zero. The bowing is also reduced, but with a monotonically decreasing rate as the silicon substrate thickness is increased to $1000 \mu \mathrm{m}$. This behaviour is readily understood at the two extremes, the composite wafer bow is governed by the stiffness of the silicon $\left(t_{S}>>t_{F}\right)$ or the stiffness of the polycrystalline diamond $\left(t_{S}<<t_{F}\right)$ at these conditions.

The results from figure 5 expose a problem likely to arise if a thick silicon substrate is to be thinned by etching or polishing after growth of the CVD polycrystalline diamond to enable optimum heat extraction of any GaN semiconductor device grown on the free silicon surface. The increase in bow as the silicon is thinned will increase the likelihood of wafer breakage owing to the build up of tensile stress in the silicon. One of the advantages of the FE model is that it can be readily extended to include the temperature dependent material properties from table 2 and table 4 as shown by open triangular data points $(\triangle)$ in figure 5 . The most significant result from the temperature dependent FE model is that it predicts a lower degree of wafer bowing in comparison to the results from the analytical and temperature independent FE models. In fact at low temperatures the CTE of diamond is lower than silicon while at higher temperatures the CTE of diamond is higher than silicon (Figure 1). This is significant because this suggests that the temperature independent models overestimate the degree of bowing. Understanding these effects is vital because it is important to engineer the degree of bow to be as small as possible. One of the ways the bowing effects have been explored using the temperature dependent FE model is by varying the thickness of the polycrystalline diamond film, as shown in figure 6.

Figure 6 shows the predicted bow of five different wafers with diamond film thicknesses between 25 and $200 \mu \mathrm{m}$. The same process of simulating the thinning of the silicon layer from 1000 to $10 \mu \mathrm{m}$ was applied to each wafer. Figure 6 reveals that the maximum bow is smaller as the polycrystalline diamond layer thickens. The temperature dependent FE model predicts that increasing the thickness of the polycrystalline diamond from 100 to $200 \mu \mathrm{m}$ approximately halves the wafer bow. The maximum bow is reduced when the polycrystalline diamond thickness is increased due to the diamond having a stiffening effect on the wafer. When the thickness of the polycrystalline diamond layer approaches $200 \mu \mathrm{m}$, the 100 $\mathrm{mm}$ diameter wafer will have a predicted maximum bow of $\sim 1 \mathrm{~mm}$ from centre to edge. The breadth of this maximum means that the wafer will not see the dramatic increases in bow associated with thinning the silicon layer of wafers with a polycrystalline diamond thickness of $\leq 100 \mu \mathrm{m}$, indicating a potentially viable design. Refering to Figure 1, the CTE of GaN is greater than both diamond and silicon. If GaN is epitaxially grown on the free silicon surface, i.e. the underside of the silicon in Figures $2 \mathrm{c}$ and $4 \mathrm{~b}$, then the higher CTE of GaN could increase bow; although the GaN device layers are expected to be relatively thin (a few microns) [27].

A final point is that the analytical and FE models explored in this paper assume that the wafer is perfectly elastic with no plastic deformation of the silicon substrate. Lattice dislocations can partially relax the wafer, reducing the overall amount of bow; however experimental measurements by Zimmer $e t$ al [5] show that on removal of the diamond by oxidation there was no observable plastic deformation of the silicon.

\section{Comparison of FE Model with Experimental Data}

The only available experimental data found in the literature for bow of silicon - polycrystalline diamond wafers is in Zimmer et al [5]. The thickness of the silicon layer was $525 \mu \mathrm{m}$ [28] and the wafer diameter was $100 \mathrm{~mm}$ [5]. The deposition layer of polycrystalline diamond was increased from 2 to 20 $\mu \mathrm{m}$ at $2 \mu \mathrm{m}$ intervals. FE models of diamond-silicon wafer were generated at three different temperatures $\left(715,755\right.$ and $\left.800^{\circ} \mathrm{C}\right)$ to compare with the experimental observations; where 715 and $755{ }^{\circ} \mathrm{C}$ are the growth temperatures used in [5]. The temperature dependent elastic and CTE data of silicon and diamond were used in the model and figure 7 indicates the model predictions. 
Zimmer et al highlighted that a cooler processing temperature (e.g. $715^{\circ} \mathrm{C}$ ) leads to a higher compressive stress in the diamond films and a higher degree of bow compared to $755^{\circ} \mathrm{C}$. It was stated that this observation did not correlate with published CTE data for diamond and silicon. However the modelling results in figure 7, where bow is only generated by CTE mismatch, clearly demonstrate that higher processing temperatures can lead to reduced bow due the CTE of diamond being larger than silicon at higher temperatures (Figure 1). It was also experimentally observed that the rate of change of stress, and bow, decreased as the diamond film becomes thicker. This is also observed in figure 7 and is related to the overall stiffness of the diamond-silicon wafer increasing with increasing diamond thickness. The absolute value of bow in the model is greater than the experimental measurements by a factor of three to four. This could be related to plastic deformation of the silicon, the diamond film exhibiting a lower elastic modulus compared to the model values or intrinsic stress within the diamond layer. For example, papers [11,12] report relatively low elastic coefficients for polycrystalline diamond due to imperfections and impurities. To examine the influence of changes in the elastic modulus of diamond on bow, the experimental data of Zimmer et al. was compared with model data for diamond grown at $715^{\circ} \mathrm{C}$ at different thicknesses on a silicon wafer $525 \mu \mathrm{m}$ thick and $100 \mathrm{~mm}$ diameter [5], figure 8. As observed in figure 7, the rate of change of bow decreases as the diamond film becomes thicker as observed with the experimental data in Figure $8(\boldsymbol{\Delta})$. The best agreement corresponds to a low diamond elastic modulus of 300GPa. Chiu et al. [29] and Kim et al. [30] demonstrated that the elastic modulus of diamond CVD films decreased considerably (from 1000 to $300 \mathrm{GPa}$ ) with increasing $\mathrm{CH}_{4} / \mathrm{H}_{2}$. Windischmann et al [31] indicated that an intrinsic and residual tensile stress can be present in diamond films grown on silicon which could also influence bow and the level of stress varies with process temperatures and $\mathrm{CH}_{4} / \mathrm{H}_{2}$ ratio [29]. Zimmer et al. reported a decrease in bow with increased diamond thickness as a result of intrinsic stress [27]; the level of the intrinsic stress can depend on quality, growth conditions, microstructure, texture and surface state (roughness) along with non-uniform heating.

\section{Orientation of silicon and diamond}

As the diamond layer grows thicker the diamond can develop an orientation in the growth direction and grains become columnar [14]. For diamond textured along [111] the modulii $1 / \mathrm{S}_{11}=1168 \mathrm{GPa}$ and $1 / \mathrm{S}_{33}$ $=1206 \mathrm{GPa}$ [32] at room temperature, which are both higher than the $1050 \mathrm{GPa}$ for polycrystalline material used in the model (see both [32] and Table 2). Finite element models were undertaken with a $100 \mathrm{~mm}$ diameter (111) silicon substrate and a $100 \mu \mathrm{m}$ diamond layer. By replacing the polycrystalline diamond with orientated diamond the maximum bow reduced from $2733 \mu \mathrm{m}$ to $2673 \mu \mathrm{m}$; the higher stiffness of the diamond restricting the wafer bow to a small extent.

While the (111) orientated silicon is the preferred substrate orientation for epitaxial GaN growth, the (100) silicon surface is also used [19]. The elastic modulus (E) and Poisson ratio (v) exhibit an isotropic character on the (111) silicon plane [23] with E=169GPa and $v=0.26$ at room temperature, see [23] and Table 4. For (100) silicon, E varies from 169GPa to 130GPa along the (100) plane and Poisson ratio varies from 0.278 to 0.062 . Based on finite element models run for (100) silicon with polycrystalline diamond the maximum bow (where the thickness of the diamond and silicon was similar) decreased from $2733 \mu \mathrm{m}$ to $2543 \mu \mathrm{m}$, which may be due to lower effective silicon stiffness limiting deformation of the diamond film (ignoring Poisson effects).

For both of these cases the changes in bow are relatively small compared to issues related to precise diamond elastic properties used due to individual growth conditions and the presence of intrinsic stress; although it seems wafer bow is more sensitive to changes in the elastic properties of the more compliant silicon substrate compared to the diamond. This may warrant a more detailed study to examine the influence of silicon and diamond orientation [32] and wafer geometry on bow. 


\section{Conclusions}

A FE model and analytical method have been developed to determine the bow of silicon-polycrystalline CVD diamond composite wafers. The model found that the maximum bow and resulting peeling moment of a particular wafer structure occurred at a point where the thicknesses of both the silicon and polycrystalline diamond layers were nearly identical, in close agreement with an analytical model. The finite element approach enabled the variation in silicon and diamond properties with temperature to be modelled, which led to a smaller predicted wafer bow when compared to models using a single linear average and allows for prediction of bow at different growth conditions. The results demonstrate that the growth of diamond on a thick silicon substrate can lead to lower levels of bow. As the silicon substrate is subsequently thinned to reduce its effective thermal resistance the bow increases considerably, especially when the thicknesses of the two materials are similar. The model also predicts experimental observations that lower processing temperatures can lead to larger degree of bow and the rate of change of bow decreases with increasing diamond thickness. Differences between model and experimental data are likely to be due to differences in the experimental conditions and materials properties/quality, such as elastic properties and the presence of intrinsic stress in the diamond layer. The modelling approach could also be used to evaluate the influence of diamond thinning on wafer bow [33]. Realising the desirable combination of high thermal conductivity and large Young's modulus of polycrystalline CVD diamond and the high crystal quality of (111) silicon as a platform for epitaxial growth of GaN for sensors and high temperature electronics will require close optimisation of the growth temperature and thicknesses of the two layers forming the composite substrates.

\section{Acknowledgements}

The research leading to these results has received funding from the European Community's Seventh Framework Programme FP7/2007-2011 under grant agreement $n^{\circ} 214610$, project MORGaN. This publication reflects only the author's views and that the Community is not liable for any use that may be made of the information contained therein.

\section{References}

[1] R. R. Reeber and K. Wang, "High Temperature Elastic Constant Prediction of Some Group IIINitrides," Internet Journal of Nitride Semiconductor Research, vol. 6, pp. 1-5, 2001.

[2] K. Kim, "Nanopatterned Surface Effect on the Epitaxial growth of InGaN/GaN Multi-quantum Well Light Emitting Diode Structure", Transactions on Electrical and Electronic Materials, vol. 10, pp. 40-43, 2009.

[3] A. Dadgar, F Schulze, M. Wienecke, A. Gadanecz, J. Bläsing, P. Veit, T. Hempel, A. Diez, J Christen and A Krost, Epitaxy of GaN on silicon-impact of symmetry and surface reconstruction, New Journal of Physics, vol. 9, pp.389(1-10), 2007.

[4] S. Joblot, F. Semond, F. Natali, P. Vennéguès, M. Laügt, Y. Cordier, and J. Massies, Growth of wurtzite-GaN on silicon (100) substrate by molecular beam epitaxy, phys. stat. sol. (c), vol. 2, pp. 2187-2190, 2005.

[5] J. W. Zimmer, G. Chandler, and T. Sharda, "Wide area polycrystalline diamond coating and stress control by sp(3) hot filament CVD reactor," Thin Solid Films, vol. 516, pp. 696-699, 2008.

[6] J. Zimmer and K.V. Ravi, Aspects of scaling CVD diamond reactors, Diamond and Related Materials, vol. 15, pp. 229-233, 2006.

[7] C. Xu, C. Wang, C. Chan, and K. Ho, "Theory of the thermal expansion of Si and diamond," Physical Review B, vol. 43, pp. 5024-5027, 1991.

[8] C. Wort and e. t. al, "Thermal Properties of bulk polycrystalline CVD diamond.," Diamond and Related Materials, vol. 3, pp. 1158-1167, 1994. 
[9] M. R. Brozel, "Thermal Properties," in Properties of Crystalline Silicon, R. Hull, Ed. London: INSPEC, 1999

[10] R.R.Reeber and K.Wang, Lattice parameters and thermal expansion of GaN, Journal of Materials Research, vol. 15 pp. 40- 44, 2000

[11] J. Wilks and E. Wilks, Properties and Applications of Diamond. New York: ButterworthHeinemann, 1991.

[12] J. E. Field, The Properties of natural and synthetic diamond: Academic Press, 1992.

[13] F. Szuecs, M. Werner, R. Sussmann, C. Pickles, and H. Fecht, "Temperature dependence of Young's modulus and degradation of chemical vapor deposited diamond," Journal of Applied Physics, vol. 86, pp. 6010-6017, 1999.

[14] P.W.May, Diamond thin films: a 21st-century material, Phil. Trans. R. Soc. Lond. A, vol. 358 pp. 473-495, 2000.

[15] D.J. Pickrell, K.A. Kline and R.E. Taylor, Thermal expansion of polycrystalline diamond produced by chemical vapor deposition, Applied Physics Letters, Vol.64, pp. 2353-2355, 1994.

[16] H. Watanabe, N. Yamada, M. Okaji, Linear thermal expansion coefficient of silicon from 293 to 1000 K, International Journal of Thermophysics, vol. 25, pp. 221-236, 2004

[17] H. McSkimin and P. Andreatch Jr, "Elastic moduli of diamond as a function of pressure and temperature," Journal of Applied Physics, vol. 43, pp. 2944-2948, 1972.

[18] C. Wort and R. Balmer, "Diamond as an electronic material," Materials Today, vol. 11, pp. 2228, 2008.

[19] R. A. Rudder, G. C. Hudson, J. B. Posthill, R. E. Thomas, and R. J. Markunas Direct deposition of polycrystalline diamond films on $\operatorname{Si}(100)$ without surface pre-treatment. App. Phys. Lett. vol. 59 pp. 7911991.

[20] C. Liu, W. N. Wang, S. Denchitcharoen, A. Gott, P. A. Shields, and D. W. E. Allsopp, "GaN nano-pendeo-epitaxy on Si (111) substrates," physica status solidi (c), vol. 6, pp. S527-S530 2009.

[21] Y. Varshni, "Temperature dependence of the elastic constants," Physical Review B, vol. 2, pp. 3952-3954, 1970.

[22] H. McSkimin, "Measurement of Elastic Constants at Low Temperatures by Means of Ultrasonic Waves - Data for Silicon and Germanium Single Crystals, and for Fused Silica," Journal of Applied Physics, vol. 24, pp. 988-997, 1953.

[23] C. H. Cho, "Characterization of Young's modulus of silicon versus temperature using a "beam deflection" method with a four-point bending fixture," Current Applied Physics, vol. 9, pp. 538$545,2009$.

[24] J. Wortman and R. Evans, "Young's modulus, shear modulus, and Poisson's ratio in silicon and germanium," Journal of Applied Physics, vol. 36, p. 153, 1965.

[25] C. Hsueh, "Thermal stresses in elastic multilayer systems," Thin Solid Films, vol. 418, pp. 182188, 2002.

[26] "ANSYS," 11.0 ed: ANSYS Inc.

[27] J.W.Zimmer, Enhancing semiconductors with diamond, Mater. Res. Soc. Symp. Proc. vol 956, pp.135-142 2007.

[28] G. Chandler, "sp3 Diamond: Wafer thickness ", personal communication M. J. Edwards, Ed., 27th October 2009.

[29] C.Chiu, Y.Liou, Y-D Juang, , Elastic modulus of and residual stresses in diamond films, Thin Solid Films vol. 260 pp. 118-123 (1995).

[30] J. G. Kim and J.Yu, Measurement of Residual Stress in Diamond Films Obtained Using Chemical Vapor Deposition, Jpn. J. Appl. Phys. vol. 37 pp. L890-L893 1998

[31] H. Windischmann, G. F. Epps, Y. Cong, and R. W. Collins, "Intrinsic stress in diamond films prepared by microwave plasma CVD," Journal of Applied Physics, vol. 69, p. 2231, 1991.

[32] E.Anastassakis and M.Siakavellas, Elastic properties of textured silicon and diamond, vol. 90 pp. 144-151, 2001.

[33] P.W.May, The New Diamond Age?, Science, vol. 319, pp.1490-1491, 2008. 



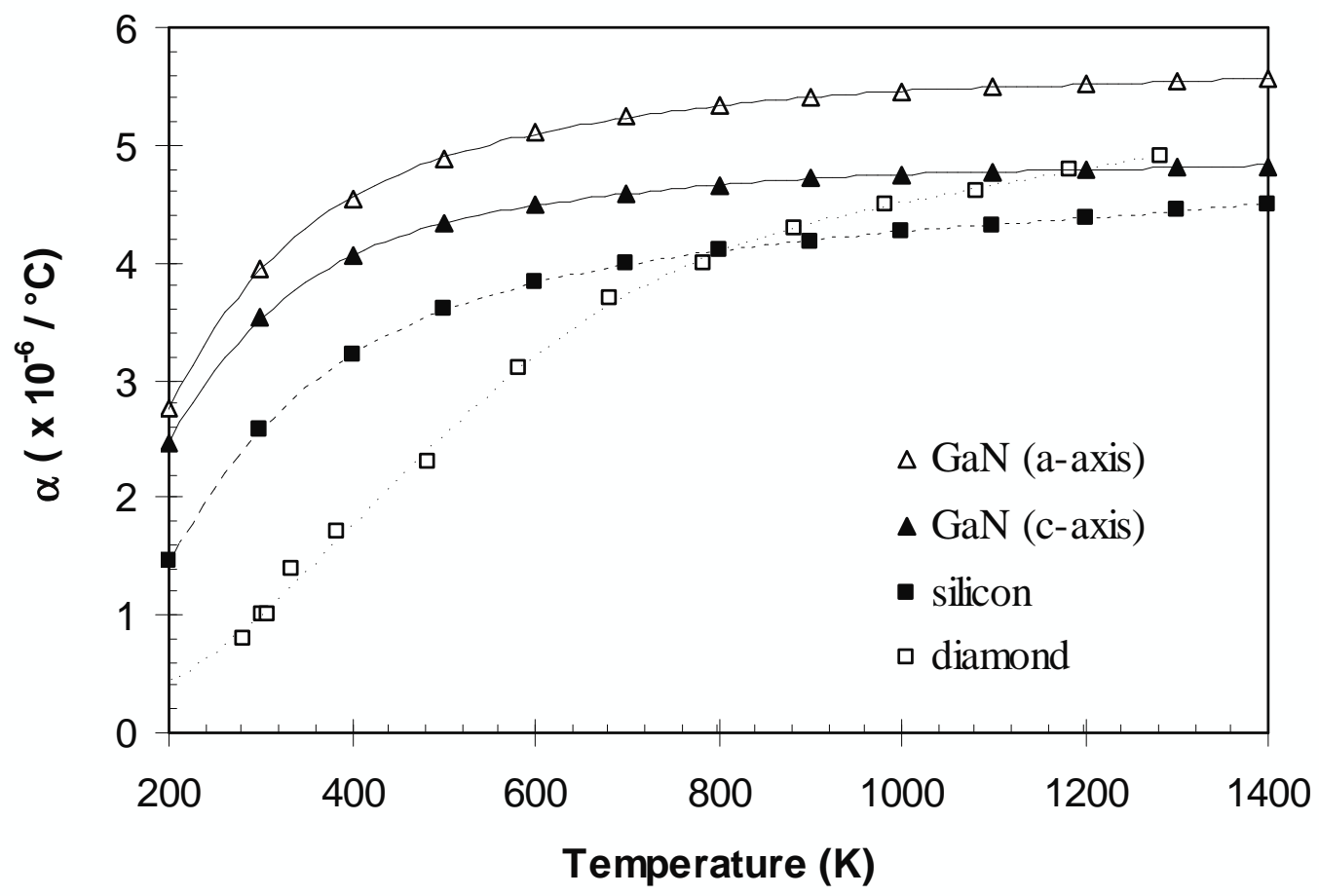

Figure 1. Thermal expansion coefficients of silicon[9], diamond [7,8] and GaN [10], 
(a)

\begin{tabular}{|c|c|c|}
\hline $\begin{array}{l}\text { CVD } \\
\text { Diamond }\end{array}$ & \multicolumn{2}{|r|}{ film layer } \\
\hline Silicon & $t_{s}$ & substrate \\
\hline
\end{tabular}

(b)

Cooling by $\Delta T$

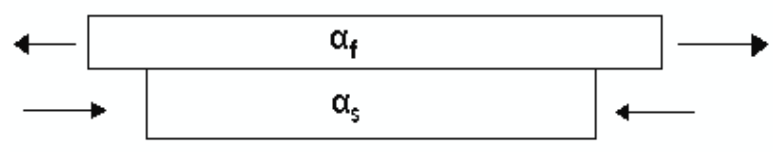

(c)

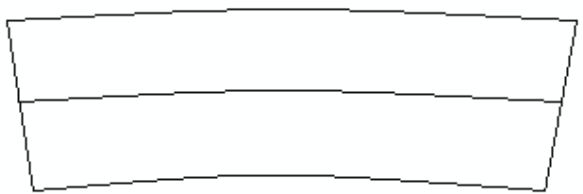

Figure 2. Bowing behaviour of the bi-layer wafer. (a) The polycrystalline CVD diamond film is grown at $800^{\circ} \mathrm{C}$ and the wafer is at thermal and structural equilibrium. (b) The wafer is cooled to room temperature and the film and substrate have contracted by differing amounts due to a mismatch in CTEs. (c) As the strain in the wafer is constrained, it bows due to asymmetric stresses [16]. 


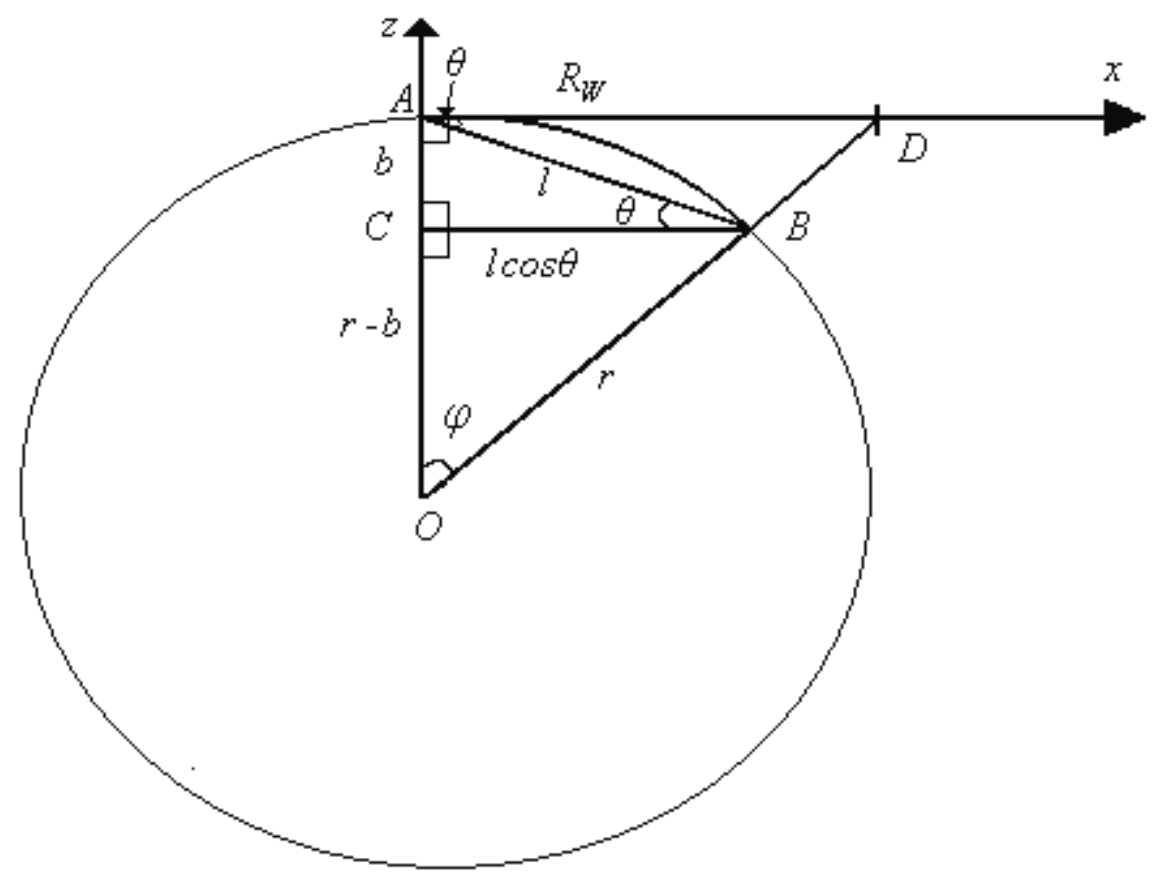

Figure 3. Geometry used to determine the wafer bow. 
Clamped edge $\Delta z=0$

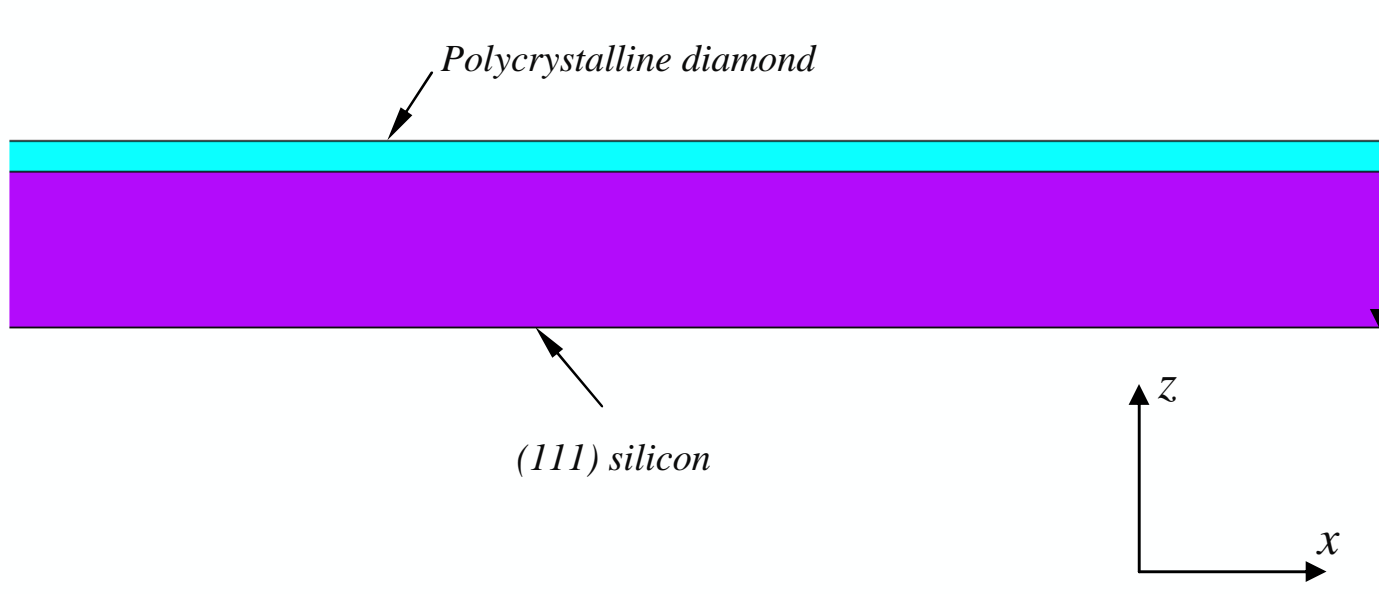

(a)
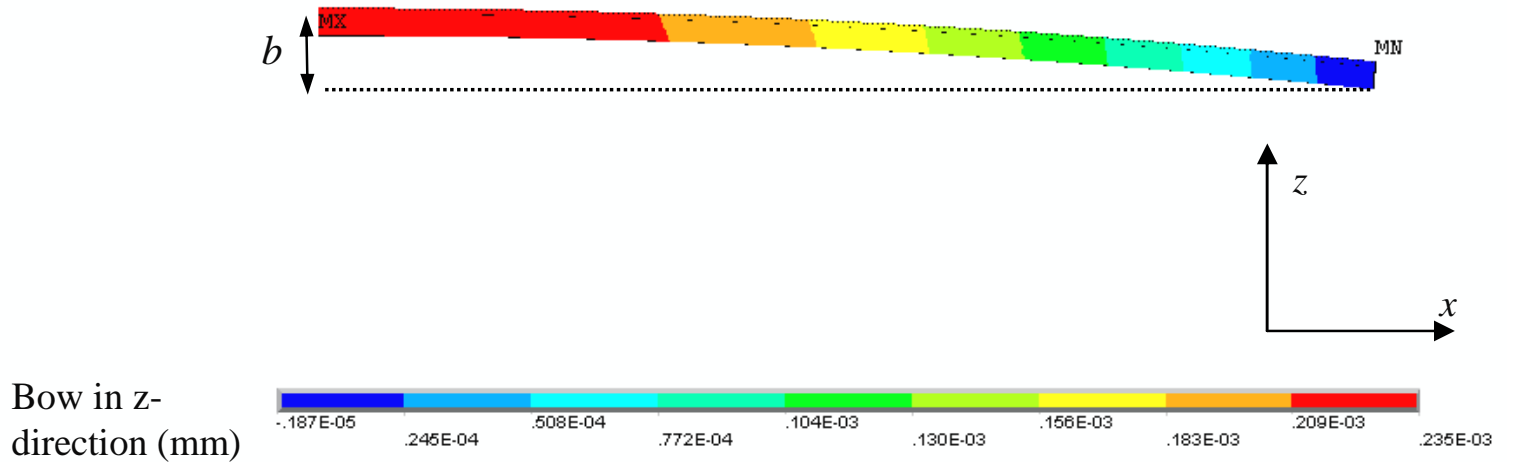

Figure 4. (a) Schematic of the wafer model. (b) FE model calculation of wafer bow, which is the displacement in the $-\mathrm{z}$ direction. 


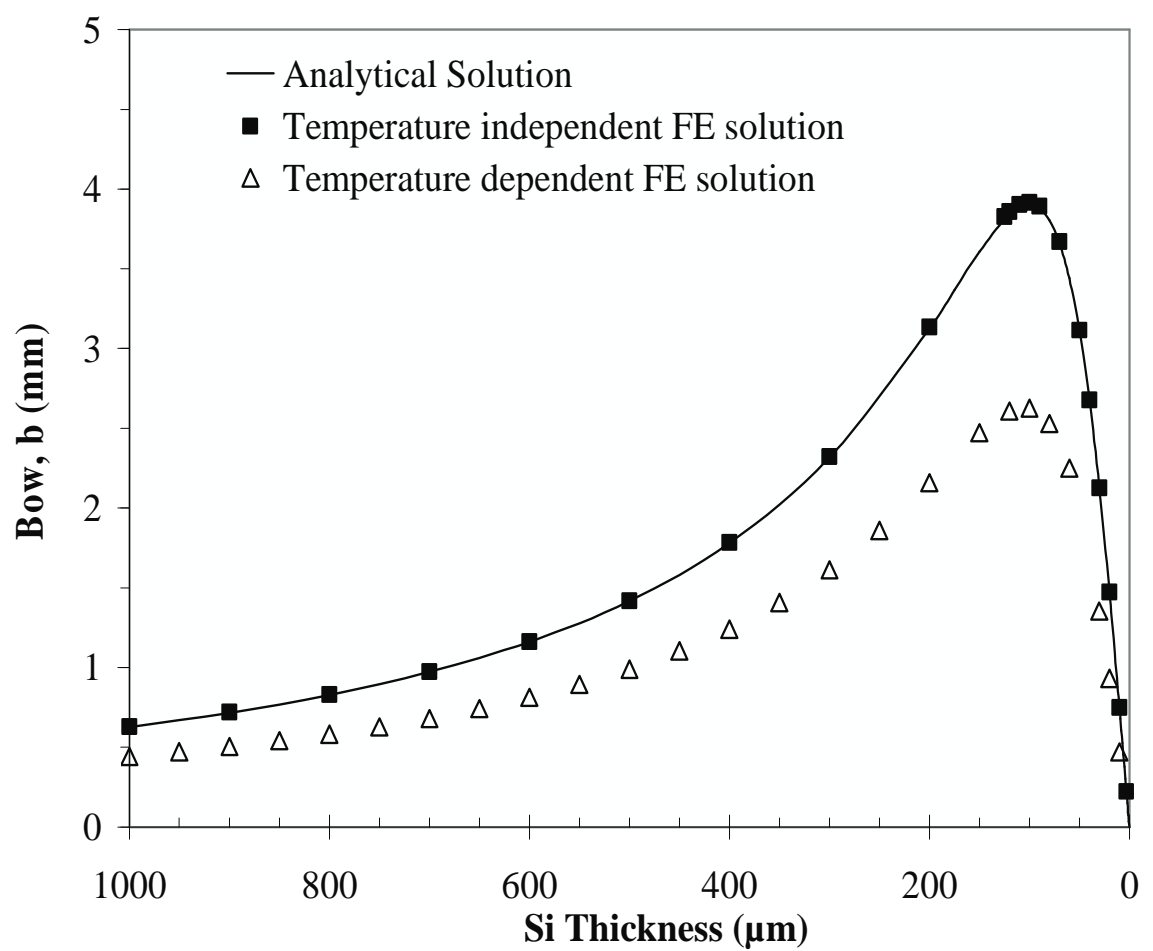

Figure 5. Graph comparing the bow measured in both the temperature independent and temperature dependent FE models with the bow predicted in the analytical solution for a $100 \mathrm{~mm}$ wafer with a $100 \mu \mathrm{m}$ thin film layer of polycrystalline CVD diamond. 


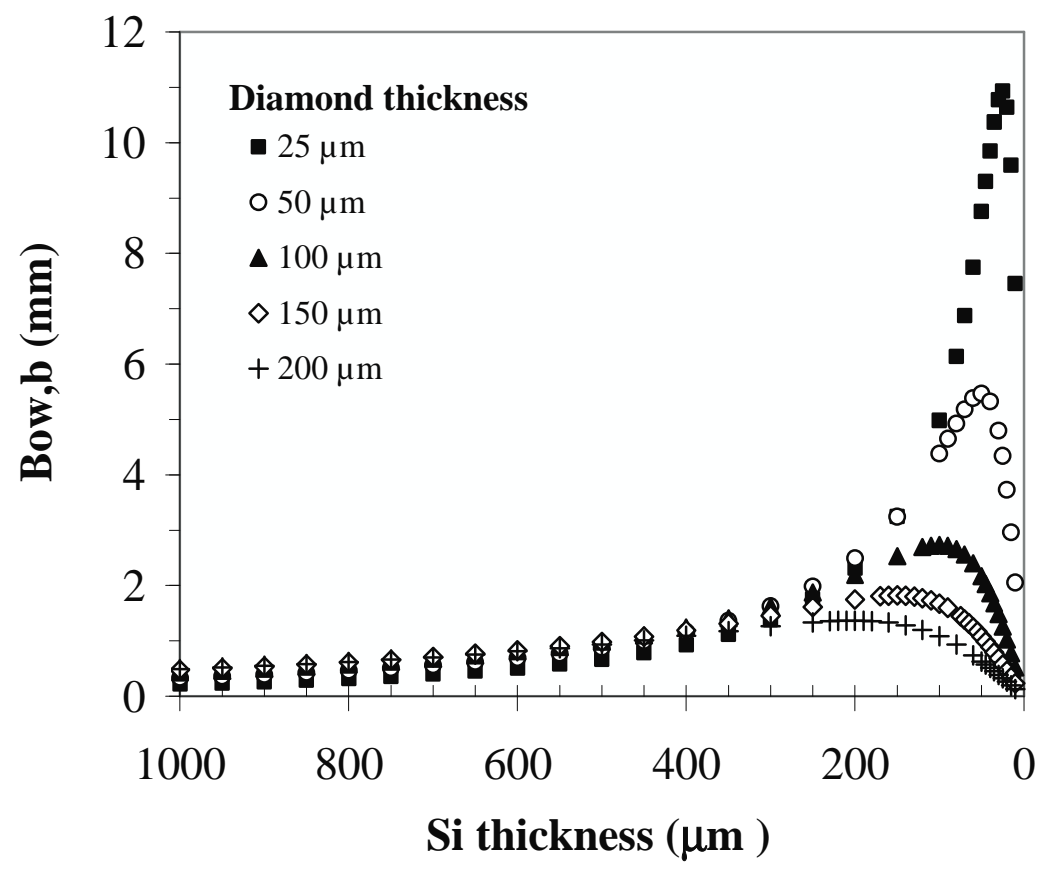

Figure 6. Wafer bow in a composite (111)Si/polycrystalline diamond wafer versus thickness of the $\mathrm{Si}$ substrate for five thicknesses of polycrystalline diamond using the temperature dependent FE model. 


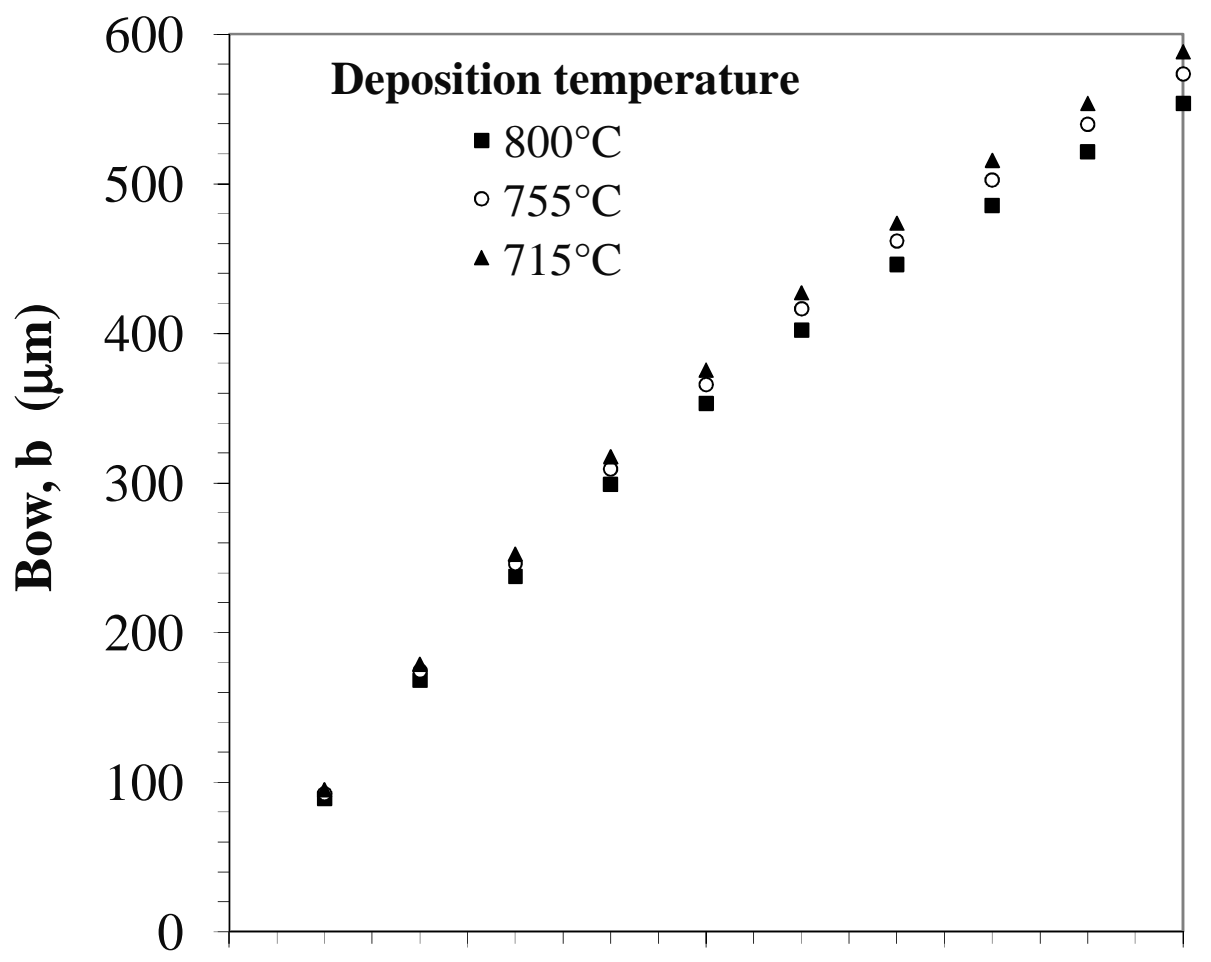

Figure 7. Wafer bow at different growth temperatures based on dimension in [2]. 


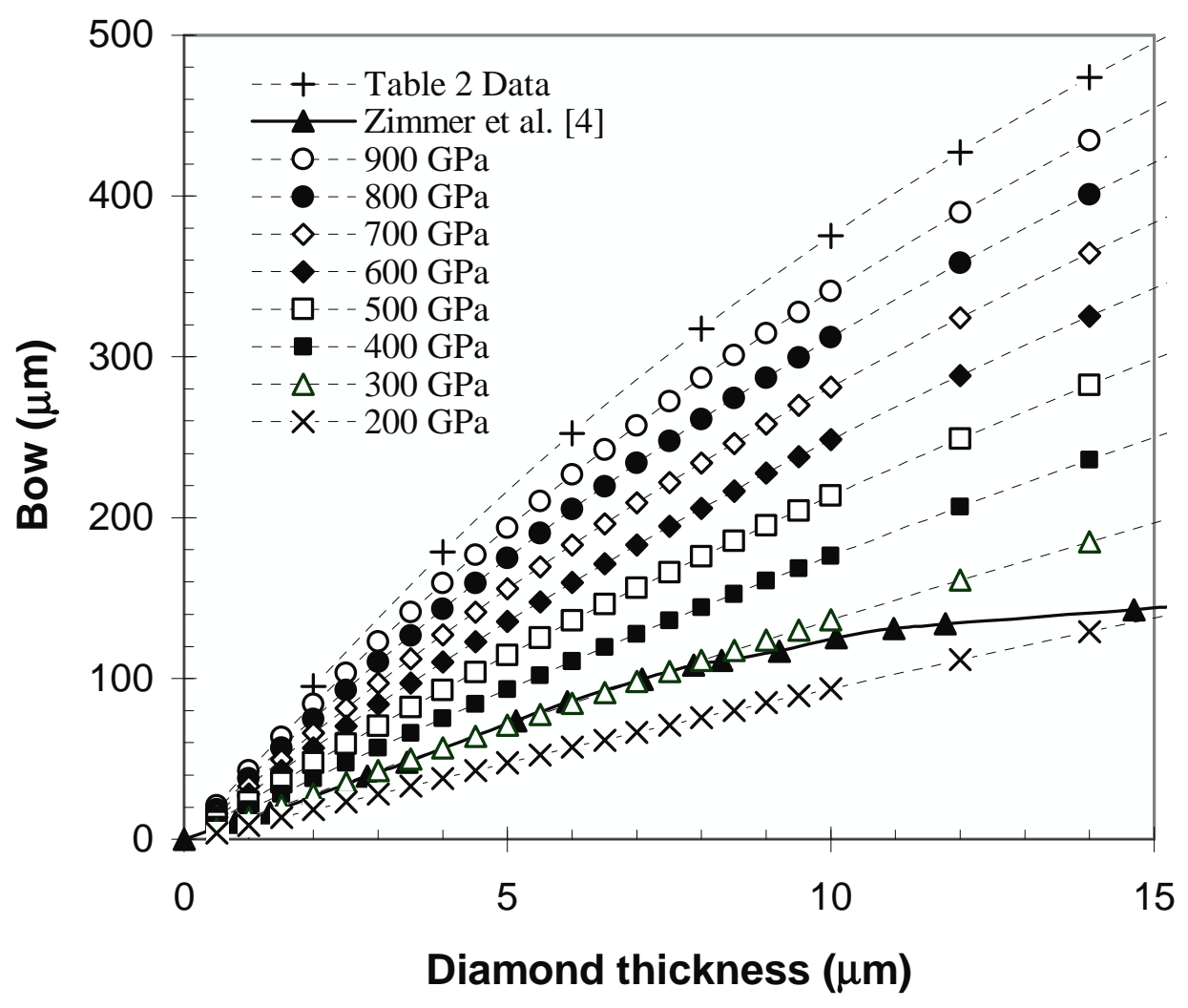

Figure 8. Bow as function of diamond stiffness for $525 \mu \mathrm{m}$ thick Si at $715^{\circ} \mathrm{C}$ 